\title{
Triple Chemotherapy (DOX) in Patients with Advanced Gastro- Esophageal Adenocarcinoma - A Phase II Study
}

\author{
${ }^{1}$ Department of Oncology, Odense University Hospital, Odense, Denmark \\ ${ }^{2}$ Department of Oncology, Aalborg Hospital, Aalborg, Denmark \\ ${ }^{3}$ Institute of Clinical Research, University of Southern Denmark, Denmark
}

Tarpgaard $L S^{1^{*}}$, Jensen $M^{2}$, Schønnemann $K R^{1}$, Jensen $H A^{1}$, Yilmaz $M^{2}$ and Pfeiffer $P^{1,3}$

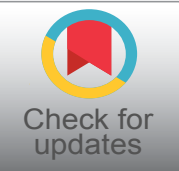

*Corresponding author: Dr. Line Schmidt Tarpgaard, Department of Oncology, Odense University Hospital, Sdr. Boulevard 29, 5000 Odense C, Denmark, Tel: +452-2511-616

\begin{abstract}
Triple chemotherapy improves efficacy in patients with advanced gastro-esophageal (GE) cancer. In a phase I dose-finding trial including 23 patients we established a recommended dose of triple chemotherapy with docetaxel $(D)$, oxaliplatin (O), and capecitabine (X) (DOX). We found promising activity and here we present efficacy data from the succeeding phase II trial. Patients and Methods: All patients had histologically confirmed GE adenocarcinoma. Therapy was docetaxel $\left(51 \mathrm{mg} / \mathrm{m}^{2}\right.$ as a 60 minutes infusion day 1$)$, oxaliplatin $\left(100 \mathrm{mg} / \mathrm{m}^{2}\right.$ as a 30 minutes infusion day 1) and capecitabine $\left(1250 \mathrm{mg} / \mathrm{m}^{2} /\right.$ day continuously). Treatment was repeated every 3 weeks until progressive disease or unacceptable toxicity. Toxicity and response were evaluated according to NCIC-CTC 3.0 and RECIST 1.0, respectively. Results: Forty-two patients with median age of 64 years and metastatic disease were included. Six patients (14\%) and 22 patients (52\%) obtained complete or partial response, respectively for an investigator evaluated response rate of $66 \%$. Median progression-free survival was 7.7 months; and overall survival was 11.8 months. Neutropenia grade 3 and 4 was observed in 20 patients (total $48 \%$ ). Febrile neutropenia was observed in 14 patients $(33 \%)$. The median number of DOX was 6. Conclusion: DOX (D: $51 \mathrm{mg} /$ $\mathrm{m}^{2}$ day 1, O: $100 \mathrm{mg} / \mathrm{m}^{2}$ day 1 and $\mathrm{X}: 1250 \mathrm{mg} / \mathrm{m}^{2}$ ) continuously every 3 weeks in patients with advanced GE cancer can be administered in an out-patient setting and efficacy is very promising and deserves evaluation in larger trials.
\end{abstract}

\section{Introduction}

Cancer of the stomach and esophagus are the fourth most frequent types of malignancies and the second most common causes of cancer related deaths worldwide [1]. Despite that treatment strategy has been so- mewhat more aggressive in recent years, patients with advanced gastro-esophageal adenocarcinomas (aGEA) still have a very poor prognosis. Several trials have confirmed that combination chemo-regimens resulted in a higher response rate (RR) than monotherapy, and a meta-analysis also demonstrated a significant and consistent survival advantage for combination chemotherapy compared to single-agent treatment [2,3]. Fluoropyrimidine- and platinum-based therapies are the backbones of treatment for aGEA. In order to reduce the side effects and make treatment easier for out-patients, several studies have investigated and found that capecitabine $(X)$ could replace fluorouracil (F) and that oxaliplatin $(O)$ could replace cisplatin (C) $[4,5]$. The REAL-2 trial [4] examined various combinations of chemotherapy. Epirubicin (E), cisplatin and 5-FU was chosen as the control arm, and cisplatin was substituted by oxaliplatin and fluorouracil by the oral capecitabine in a $2 \times 2$ factorial design. The authors found non-inferiority of both capecitabine and oxaliplatin in the two-bytwo comparison (primary endpoint) but also a longer overall survival (OS) in the EOX group than in the ECF group, with a median OS of 11.2 months. Subsequently, a number of trials have confirmed the efficacy of capecitabine and oxaliplatin $[6,7]$. Furthermore, studies suggested that triple chemotherapy improves efficacy in patients with aGEA. The addition of an anthracycline or a taxane to a platinum and a fluoropyrimidine has resulted in higher RR and a modest improvement in OS compared with doublet combinations, but it also expo-

Citation: Tarpgaard LS, Jensen M, Schønnemann KR, Jensen HA, Yilmaz M, et al. (2021) Triple Chemotherapy (DOX) in Patients with Advanced Gastro-Esophageal Adenocarcinoma - A Phase II Study. Int J Cancer Clin Res 8:147. doi.org/10.23937/2378-3419/1410147

Accepted: March 17, 2021: Published: March 19, 2021

Copyright: (C) 2021 Tarpgaard LS, et al. This is an open-access article distributed under the terms of the Creative Commons Attribution License, which permits unrestricted use, distribution, and reproduction in any medium, provided the original author and source are credited. 
ses patients to more serious side effects $[2,3,8,9]$. In the V-325 trial [8] patients were treated with cisplatin and fluorouracil with or without docetaxel as a first-line therapy. RR, progression-free survival (PFS), and two-years OS were improved by the addition of docetaxel. Several studies and meta-analysis have confirmed the efficacy of taxanes [2,10-13] and a taxane-containing triple combination is presently the most promising regimen but the most optimal schedule is not established.

In view of the significant toxicities associated with the triple taxane-containing regimen, several modified regimens have been developed. In a phase I dose finding trial including 23 patients [14] we established a recommended dose of triple chemotherapy with docetaxel, short-time infusion of oxaliplatin, and continuously capecitabine (DOX). We found promising activity and here we present efficacy data from the succeeding phase II trial.

\section{Patients and Methods}

All patients were required to have histologically confirmed adenocarcinoma of the lower oesophagus, the gastro-oesophageal junction or the stomach and not availed for surgical resection. Other criteria for inclusion were measurable disease according to RECIST 1.0, WHO performance status (WHO PS) 0-1, age over 18 years, no prior chemotherapy other than adjuvant chemotherapy completed at least six months before inclusion, adequate bone marrow function (neutrophils count $>1.5 \times 10^{9} / \mathrm{l}$; platelets $>100 \times 10^{9} / \mathrm{l}$ ), adequate hepatic function (serum bilirubin $\leq 1.5 \times$ upper normal limit (UNL), transaminases $\leq 3 \times$ UNL (however, in cases of liver metastases there were no upper limit for transaminases)), adequate renal function (calculated creatinine clearance $\geq 60 \mathrm{ml} \mathrm{min}^{-1}$ by the Cockroft and Gault formula). Furthermore, the treatment should be started within eight days after inclusion, and the patients may not have peripheral neuropathy, any co-existing severe medical illness, sign of brain metastases or receive concomitant treatment with other anticancer drugs. Female were not included if they were pregnant or lactating.

The study was approved by the local ethics committee and the Danish Health Authority (Eudract nr. 2006002270-21). Signed informed consents were obtained from all patients before entering the study.

\section{Treatment schedule}

Therapy was initiated with docetaxel $\left(60 \mathrm{mg} / \mathrm{m}^{2}\right.$ as a 60 minutes infusion day 1), oxaliplatin $\left(115 \mathrm{mg} / \mathrm{m}^{2}\right.$ as a 30 minutes infusion day 1$)$, and capecitabine (1250 mg/ $\mathrm{m}^{2} /$ day continuously) as recommended from our phase I study [14]. In order to maintain treatment cadence without dose reductions and hopefully lesser toxicity we amended the phase II protocol and reduced the dose with $15 \%$ of docetaxel to $51 \mathrm{mg} / \mathrm{m}^{2}$ and of oxaliplatin to $100 \mathrm{mg} / \mathrm{m}^{2}$ [15]. The new recommendation was amended to and approved by the local ethics committee and the Danish Health Authority and was used after 12 patients had received the higher dose of DOX. DOX was repeated every 3 weeks for a planned number of 8 cycles.

\section{Assessment}

Before inclusion, each patient was assessed by complete physical examination, full blood count, clotting profile, blood biochemistry, 12-lead electrocardiogram, contrast-enhanced CT scan of the thorax, abdomen, and pelvis, and a pregnancy test for women with child-bearing potential. Subsequently, complete physical examination, blood biochemistry, and a toxicity and adverse event assessment were repeated before each cycle began; a full blood count was repeated before every docetaxel infusion. From May 2010 a HER2 test was performed, and thence only HER2 negative patients were included. A tumour marker assessment and contrast-enhanced CT scan of the thorax, abdomen, and pelvis were repeated at the end of every third treatment cycle until disease progression. The response was evaluated by the investigator according to RECIST 1.0 every. Toxicity was evaluated according to NCIC-CTC 3.0.

\section{Study end-points}

The primary clinical end-point of the study was RR. Secondary end-points were OS, PFS, treatment-related toxicity, and disease-associated symptoms. PFS was defined as the time from inclusion to progressive disease occurred (according to the RECIST criteria) or death of any cause. OS was defined as the time from inclusion to death of any cause. Data was updated December $1^{\text {st }}$, 2020.

\section{Statistical analyses}

Non-parametric statistics were applied. All median values are followed by the range in brackets. After cessation of treatment patients without documented progression were followed every 3 months with clinical and radiological evaluation. The number of evaluable patients is based on Simons two-stage design [16]. PFS and OS were generated according to the Kaplan-Meier method. Data were recorded and analysed in a Medlogdatabase. All analyses were done on an intention-to-treat population.

\section{Results}

\section{Patients characteristics}

Baseline demographic and patient characteristics are summarized in Table 1. From September 2009 to September 2012 we included 42 patients, 36 males and 6 females with a median age of 64 years (IQR 44-76) and WHO PS score of 0 and 1 in $57 \%$ and $43 \%$, respectively were analysed in the intension to treat population. The primary tumour site was the lower oesophagus in 12 patients, the gastro-oesophageal junction in 23 patients and stomach in 7 patients. All of the patients had non-resectable locally advanced $(n=3)$ or metastatic disease. 
Table 1: Baseline characteristics for 42 patients with advanced gastro-esophageal adenocarcinoma treated with DOX.

\begin{tabular}{|c|c|}
\hline Number of patients & 42 \\
\hline \multicolumn{2}{|l|}{ Age, years } \\
\hline Median & 64 \\
\hline range & $44-76$ \\
\hline \multicolumn{2}{|l|}{ Sex } \\
\hline Male & $36(86 \%)$ \\
\hline Female & $6(14 \%)$ \\
\hline \multicolumn{2}{|l|}{ WHO performance status } \\
\hline 0 & $24(57 \%)$ \\
\hline 1 & $18(43 \%)$ \\
\hline \multicolumn{2}{|l|}{ Primary tumour site } \\
\hline Esophagus & $12(28 \%)$ \\
\hline GEJ & $23(55 \%)$ \\
\hline Stomach & $7(17 \%)$ \\
\hline \multicolumn{2}{|l|}{ Status of primary tumour } \\
\hline R0 resection & $5(12 \%)$ \\
\hline $\mathrm{R} 2$ resection & $1(2 \%)$ \\
\hline No surgery & $36(86 \%)$ \\
\hline \multicolumn{2}{|l|}{ No patient had prior CT or RT } \\
\hline \multicolumn{2}{|l|}{ Stage } \\
\hline Locally advanced & $3(7 \%)$ \\
\hline Metastatic & $39(93 \%)$ \\
\hline \multicolumn{2}{|l|}{ No. of organs involved } \\
\hline 1 & $2(5 \%)$ \\
\hline 2 & $14(36 \%)$ \\
\hline 3 & $14(36 \%)$ \\
\hline$\geq 4$ & $9(23 \%)$ \\
\hline \multicolumn{2}{|l|}{ Increased } \\
\hline Alkaline phosphatase (> $300 \mathrm{U} / \mathrm{l})$ & $4(10 \%)$ \\
\hline ALAT (> $40 \mathrm{U} / \mathrm{l})$ & $6(14 \%)$ \\
\hline Platelets $\left(>400 \times 10^{9} / \mathrm{I}\right)$ & $14(33 \%)$ \\
\hline ANC $\left(>7.5 \times 10^{9} / \mathrm{l}\right)$ & $22(52 \%)$ \\
\hline
\end{tabular}

\section{Administered treatments}

A total number of 252 cycles were administered in the study, with a median number of 6 cycles per patient (IQR 4-8). The cumulative doses $\left(\mathrm{mg} / \mathrm{m}^{2}\right)$ of docetaxel and oxaliplatin were $308 \mathrm{mg} / \mathrm{m}^{2}$ and $426 \mathrm{mg} / \mathrm{m}^{2}$, respectively. The median relative dose intensities (RDI) for docetaxel and oxaliplatin were 0.97 and 0.96, respectively. Reasons for discontinuation of study treatment were PD $(n=11.26 \%)$, toxicity $(n=17.41 \%)$ and completion of planned therapy $(n=14.33 \%)$. The administered treatments are shown in Table 2.

\section{Toxicity}

Treatment was generally well tolerated. Neutropenia grade 3 and 4 was observed in 20 patients (total 48\%),
Table 2: Efficacy data and dose administration for 42 patients with advanced gastro-esophageal adenocarcinoma treated receiving DOX.

\begin{tabular}{|c|c|}
\hline Number of patients & 42 \\
\hline \multicolumn{2}{|l|}{ Efficacy } \\
\hline Complete response (CR), n (\%) & $6(14 \%)$ \\
\hline Partial response (PR), n (\%) & $22(52 \%)$ \\
\hline Disease control $\left(\mathrm{CR}+\mathrm{PR}+\mathrm{NC} \mathrm{C}^{*}\right), \mathrm{n}(\%)$ & $35(83 \%)$ \\
\hline PFS, months (median) & $7.7(5.5-9.7)$ \\
\hline OS, months (median) & $11.8(7.6-15.2)$ \\
\hline \multicolumn{2}{|l|}{ Reason for discontinuation of DOX } \\
\hline Completed planned number of DOX & $14(33 \%)$ \\
\hline Progressive Disease & $11(26 \%)$ \\
\hline Toxicity or patients wish & $17(41 \%)$ \\
\hline \multicolumn{2}{|l|}{ Dose administration } \\
\hline Number of DOX (median, IQR) & $6(4-8)$ \\
\hline Number of docetaxel cycles (median, IQR) & $6(4-8)$ \\
\hline Cumulative dose of $D$, (median, IQR) & $308(204-350)$ \\
\hline Dose intensity of $D$ & $97(94-100)$ \\
\hline Cumulative dose of $O$, (median, IQR) & $426(305-592)$ \\
\hline Dose intensity of $O$, (median, IQR) & $96(82-100)$ \\
\hline Duration of therapy, months (median, IQR) & $4.8(3.0-5.5)$ \\
\hline
\end{tabular}

*NC = no change

no thrombocytopenia grade 3 or 4 was seen. Eight of 12 patients who received high dose DOX developed febrile neutropenia (66\%), but only 6 of 30 patients (20\%) after the $15 \%$ dose reduction of docetaxel and oxaliplatin. The frequencies of hematological and non-hematological adverse events are shown in Table 3. The most important non-hematologic grade 3 and 4 toxicities were neuropathy $(17 \%)$, diarrhoea (14\%), fatigue $(12 \%)$, and nausea $(7 \%)$.

\section{Efficacy}

The response rate was $66 \%$ (6 patients obtained complete response (CR) and 22 patients obtained partial response (PR)) and disease control rate was $83 \%$ (Table 2). Median PFS and OS were 7.7 months; $95 \% \mathrm{CI}$ 5.5-9.7 months and 11.8 months; $95 \% \mathrm{Cl}$ 7.6-15.2 months, respectively (Figure 1 ). Three patients with locally advanced disease died after 2, 15 and 29 months, respectively. There was no sign of difference in efficacy (RR, PFS or OS) between patients receiving the initial higher dose of DOX and the recommended adjusted DOX.

\section{Discussion}

Palliative chemotherapy prolongs OS and improves quality of life - also for older patients, but older and frail patients are underrepresented in clinical trials. The median OS in large randomized trials are approaching 12 months but in unselected populations the median OS is much lower $[17,18]$. The median age for patients with aEGA is around 70 years but patients in many recent 
Table 3: Worst toxicity for 42 patients with advanced gastro-esophageal adenocarcinoma treated receiving DOX.

\begin{tabular}{|l|l|l|l|}
\hline Hematologictoxicity & $\begin{array}{l}\text { Grade II } \\
\mathbf{n}(\%)\end{array}$ & $\begin{array}{l}\text { Grade III } \\
\mathbf{n}(\%)\end{array}$ & $\begin{array}{l}\text { Grade IV } \\
\mathbf{n}(\%)\end{array}$ \\
\hline Neutropenia & $3(7 \%)$ & $0(0 \%)$ & $10(24 \%)$ \\
\hline Thrombocytopenia & 0 & $13(31 \%)$ & $0(0 \%)$ \\
\hline Febrile neutropenia & - & $\begin{array}{l}\text { Grade III } \\
\mathbf{n}(\%)\end{array}$ & $1(2 \%)$ \\
\hline Non-hematologic toxicity & $\begin{array}{l}\text { Grade II } \\
\mathbf{n}(\%)\end{array}$ & $3(7 \%)$ & $\begin{array}{l}\text { Grade IV } \\
\mathbf{n}(\%)\end{array}$ \\
\hline Nausea & $8(19 \%)$ & 0 & 0 \\
\hline Diarrhoea & $6(14 \%)$ & $6(14 \%)$ & 0 \\
\hline Vomit & $4(10 \%)$ & $1(2 \%)$ & 0 \\
\hline Neuropathy & $13(31 \%)$ & $7(17 \%)$ & 0 \\
\hline Fatigue & $23(55 \%)$ & $5(12 \%)$ & - \\
\hline Nail toxicity & $13(31 \%)$ & $5(12 \%)$ & \\
\hline HFS & $8(19 \%)$ & $3(7 \%)$ & \\
\hline Anorexia & $3(7 \%)$ & $2(5 \%)$ & 0 \\
\hline
\end{tabular}

In addition one patient had pulmonary embolism after the $6^{\text {th }}$ cycle of DOX.

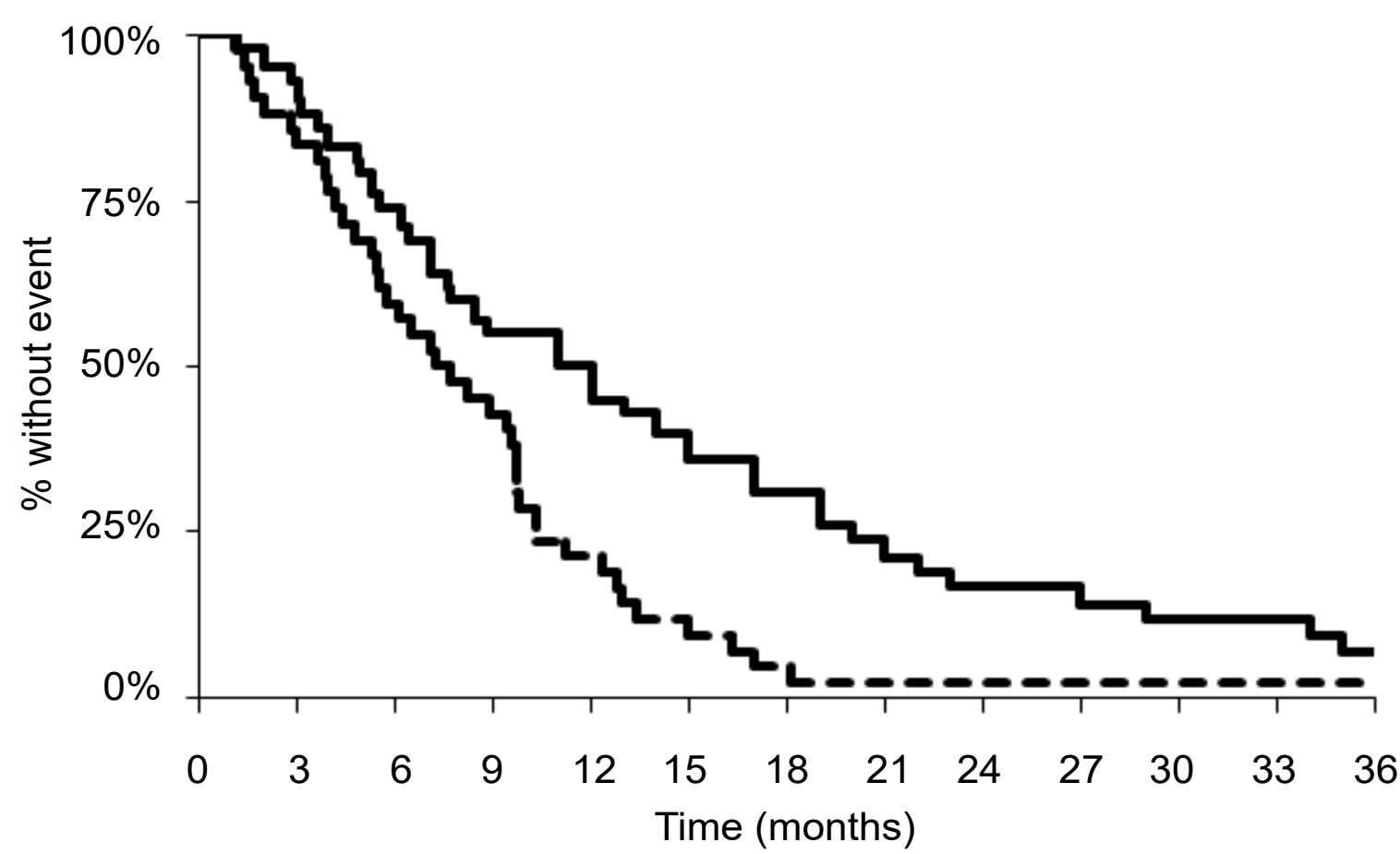

Figure 1: Kaplan-Meier survival curve demonstrating progression-free survival (dotted line) and overall survival (solid line). Kaplan-Meier curves of progression-free survival (median 7.7 months; 95\% Cl 5.5-9.7 months), dotted line, and overall survival (median 11.8 months; 95\% Cl 7.6-15.2 months), solid line.

trials are highly selected and the median age is often only around 60 years. A number of different first-line regimens have been validated for use in this setting, but there is as yet no consensus recommendation on a worId-wide combination. A combination of a platinum based chemotherapy (cisplatin or oxaliplatin) and a fluoropyrimidine (fluorouracil, capecitabine or S-1) represents the cornerstone of first line treatment $[2,3,9,19,20]$. The addition of docetaxel to such doublets improves OS further, albeit at the cost of increased toxicity. In the V325 trial [8], 455 patients across 72 centres and 16 countries, were randomized to triple DCF (docetaxal, cisplatin and fluorouracil) or CF. All efficacy parameters significantly favoured DCF over CF alone, with a higher RR (37\% Vs. 25\%), longer PFS (5.6 Vs. 3.7 months) and longer median OS (9.2 Vs. 8.6 months). Even though DCF resulted in an increased risk of severe adverse events, especially febrile neutropenia and/or neutropenic infection (29\% Vs. $12 \%$ ) and diarrhoea (19\% Vs. $8 \%$ ), preservation of quality of life and clinical benefit favoured DCF over CF $[21,22]$. Several other studies and meta-analyses have confirmed the efficacy of taxanes $[10,11,13,23,24]$ and a taxane-containing triple combination is presently the most promising regimen but the most optimal schedule 
has not been established: Therapy every second or third week; oxaliplatin or cisplatin; infusion of fluorouracil or oral alternatives and dose of docetaxel are all important topics still to be solved $[9,25]$. Recent updates conclude that three drug regimens yield a significant OS benefit compared to a two-drug regimen but at the cost of increased toxicity $[9,26]$.

Based on our phase I dose finding study [14], the present phase II study investigates the combination of DOX in patients with aGEA. The efficacy results of the current study, with a RR at $66 \%$, PFS 7.7 months and OS 11.8 months are in line with other western phase II trials [23,24,2729] with reported median PFS from 5.2 to 6.9 months and OS ranging from 8.4 to 18 months. In the present study a dose modification was necessary due to unacceptable toxicity in terms of febrile neutropenia. Eight of 12 patients who received high dose DOX developed febrile neutropenia (66\%), but only 6 of 30 patients (20\%) after the $15 \%$ dose reduction of docetaxel and oxaliplatin. This dose modification had no impact on the efficacy of the treatment. Due to the heavy haematological toxicity of the triple regimen, several authors have modified the regimen including splitted or reduced dose of all or some of the three drugs, with manageable toxicity and satisfactory results [26]. In particular, Shah, et al. [11] performed a phase II randomized study comparing standard docetaxel, cisplatin and 5-FU (DCF) regimen with modified DCF. Despite the use of granulocyte colony-stimulating factor (G-CSF), the standard DCF arm was closed prematurely due to excessive toxicity. In the present study the patients were not routinely offered G-CSF. The latest ESMO Clinical Practice guidelines [30] recommend the use of G-CSF prophylactically if the risk of febrile neutropenia is more than $20 \%$ for all planned cycles of treatment. If the patients in the present study were offered G-CSF some of them would probably avoid febrile neutropenia. In addition to providing G-CSF a change in the treatment schedule may also reduce the side-effects as seen in the study by Shah, et al. [11], where the modified schedule provide treatment every second week.

The combination of the chemotherapeutic agents is also important compared to the side-effects. A recent randomized phase II trial addressed whether 5-FU can be replaced by capecitabine in a three-drug regimen including docetaxel and oxaliplatin [24]. The patients were randomized to docetaxel + oxaliplatin (DO); docetaxel, oxaliplatin and 5-FU (DOF); or docetaxel, oxaliplatin and capecitabine (DOX). DOX was given at the same dose as in the present study. The authors found higher efficacy and less risk of febrile neutropenia in patients receiving treatment every 2 weeks. However, it needs to be confirmed whether this is caused by a slightly lower dose of docetaxel administered every 2 weeks or combination with 5-FU. When 5-FU infusion is replaced by oral therapy, capecitabine or S-1 is often administered for 14 days every 3 weeks, but a 2 weeks schedule of docetaxel, oxaliplatin and S-1 is tolerable and promising [25].
No large Western randomized study (more than 100 patients per treatment arm) has ever shown a median OS of more than 12 months and it is not expected to change dramatically with the currently available and approved cytotoxic drugs. Therefore but also inspired by results from other primaries, there has been a huge interest in the combination of biological agents with the best chemotherapeutic regimens. At present, the most promising strategy is found in patients with overexpression of HER2. Trastuzumab is well tolerated and can actually be combined with triple docetaxel regimens with very promising results [31].

In conclusion DOX (D $51 \mathrm{mg} / \mathrm{m}^{2}$ day 1 , $0100 \mathrm{mg} / \mathrm{m}^{2}$ day 1 and $X 1250 \mathrm{mg} / \mathrm{m}^{2}$ ) continuously every 3 weeks in patients with advanced gastro-esophageal cancer can be administered in an out-patient setting and efficacy is very promising and deserves evaluation in larger trials.

\section{Acknowledgement}

We want to thank all patients who participated in this trial, all institutions who included patients, and all the staff engaged in this study.

\section{Conflicts of Interest}

Per Pfeiffer has received research funding from Taiho. All other authors declare no conflict of interest. The authors alone are responsible for the content and writing of the paper.

\section{Role of the Funding Source}

Financial support from Roche $A / S$ to salaries for project nurse.

Roche A/S had no role in the design and conduct of the study; in the collection, management, analysis, and interpretation of the data; or in the preparation, review, or approval of the manuscript.

\section{References}

1. Bray F, Ferlay J, Soerjomataram I, Siegel RL, Torre LA, et al. (2018) Global cancer statistics 2018: GLOBOCAN estimates of incidence and mortality worldwide for 36 cancers in 185 countries. CA Cancer J Clin Nov 68: 394-424.

2. Wagner AD, Unverzagt S, Grothe W, Kleber G, Grothey A, et al. (2010) Chemotherapy for advanced gastric cancer. Cochrane Database Syst Rev 8: CD004064.

3. Lordick F, Lorenzen S, Yamada Y, Ilson D (2014) Optimal chemotherapy for advanced gastric cancer: Is there a global consensus? Gastric Cancer 17: 213-225.

4. Cunningham $D$, Starling $N$, Rao $S$, Iveson $T$, Nicolson $M$, et al. (2008) Capecitabine and oxaliplatin for advanced esophagogastric cancer. N Engl J Med 358: 36-46.

5. Kang YK, Kang WK, Shin DB, Chen J, Xiong J, et al. (2009) Capecitabine/cisplatin versus 5 fluorouracil/cisplatin as first-line therapy in patients with advanced gastric cancer: A randomised phase III noninferiority trial. Ann Oncol 20: 666-673.

6. Al-Batran SE, Hartmann JT, Probst S, Schmalenberg H, Hollerbach S, et al. (2008) Phase III trial in metastatic ga- 
stroesophageal adenocarcinoma with fluorouracil, leucovorin plus either oxaliplatin or cisplatin: A study of the Arbeitsgemeinschaft Internistische Onkologie. J Clin Oncol 26: 1435-1442.

7. Schonnemann KR, Jensen HA, Yilmaz M, Jensen BY, Larsen O, et al. (2008) Phase II study of short-time oxaliplatin, capecitabine and epirubicin (EXE) as first-line therapy in patients with non-resectable gastric cancer. $\mathrm{Br} \mathrm{J}$ Cancer 99: 858-861.

8. Van Cutsem E, Moiseyenko VM, Tjulandin S, Majlis A Constenla M, et al. (2006) Phase III study of docetaxel and cisplatin plus fluorouracil compared with cisplatin and fluorouracil as first-line therapy for advanced gastric cancer: a report of the V325 Study Group. J Clin Oncol 24: 49914997.

9. Ter Veer E, Haj Mohammad N, van Valkenhoef G, Ngai LL, Mali RM, et al. (2016) The Efficacy and Safety of First-line Chemotherapy in Advanced Esophagogastric Cancer: A Network Meta-analysis. J Natl Cancer Inst 108.

10. Wang J, Xu R, Li J, Bai Y, Liu T, et al. (2016) Randomized multicenter phase III study of a modified docetaxel and cisplatin plus fluorouracil regimen compared with cisplatin and fluorouracil as first-line therapy for advanced or locally recurrent gastric cancer. Gastric Cancer 19: 234-244.

11. Shah MA, Janjigian YY, Stoller R, Shibata S, Kemeny M, et al. (2015) Randomized Multicenter Phase II Study of Modified Docetaxel, Cisplatin, and Fluorouracil (DCF) Versus DCF Plus Growth Factor Support in Patients With Metastatic Gastric Adenocarcinoma: A Study of the US Gastric Cancer Consortium. J Clin Oncol 33: 3874-3879.

12. Van Cutsem E, Lenz HJ, Kohne CH, Heinemann V, Tejpar S, et al. (2015) Fluorouracil, leucovorin, and irinotecan plus cetuximab treatment and RAS mutations in colorectal cancer. J Clin Oncol 33: 692-700.

13. Wagner AD MM, Grothe W, Haerting J, Unverzagt S (2015) Novel chemotherapy combinations for metastatic gastric adenocarcinoma (mAGC): An updated meta-analysis. J Clin Oncol 33.

14. Andersen M, Schonnemann KR, Yilmaz M, Jensen $H A$, Vestermark LW, et al. (2010) Phase I study of docetaxel, oxaliplatin and capecitabine (TEX) as first line therapy to patients with advanced gastro-oesophageal cancer. Acta Oncol 49: 1246-1252.

15. Schonnemann KR, Yilmaz $M$, Andersen $M$, Pfeiffer $P$ (2011) New recommendation of doses in an ongoing phase II study of docetaxel, oxaliplatin and capecitabine as first line therapy in advanced gastro-oesophageal cancer. Acta Oncol 50: 151-152.

16. Simon R (1989) Optimal two-stage designs for phase II clinical trials. Control Clin Trials 10: 1-10.

17. Schonnemann KR, Mortensen MB, Bjerregaard JK, Fristrup C, Pfeiffer P (2014) Characteristics, therapy and outcome in an unselected and prospectively registered cohort of patients with gastro-oesophageal cancer. Acta Oncol 53: 385-391.

18. Yang D, Hendifar A, Lenz C, Togawa K, Lenz F, et al (2011) Survival of metastatic gastric cancer: Significance of age, sex and race/ethnicity. J Gastrointest Oncol 2: 77-84.

19. Waddell T, Verheij M, Allum W, Cunningham D, Cervantes A, et al. (2014) Gastric cancer: ESMO-ESSO-ESTRO Clinical Practice Guidelines for diagnosis, treatment and follow-up. Radiother Oncol 110: 189-194.

20. Ajani JA, Bentrem DJ, Besh S, D'Amico TA, Das P, Denlin- ger C, et al. (2013) Gastric cancer, version 2.2013: Featured updates to the NCCN Guidelines. J Natl Compr Canc Netw 11: 531-546.

21. Ajani JA, Correa AM, Swisher SG, Wu TT (2007) For localized gastroesophageal cancer, you give chemoradiation before surgery, but then what happens? J Clin Oncol 25: 4315-4316.

22. Ajani JA, Moiseyenko VM, Tjulandin S, Majlis A, Constenla $M$, et al. (2007) Quality of life with docetaxel plus cisplatin and fluorouracil compared with cisplatin and fluorouracil from a phase III trial for advanced gastric or gastroesophageal adenocarcinoma: The V-325 Study Group. J Clin Oncol 25: 3210-3216.

23. Al-Batran SE, Pauligk C, Homann N, Hartmann JT, Moehler M, et al. (2013) The feasibility of triple-drug chemotherapy combination in older adult patients with oesophagogastric cancer: A randomised trial of the Arbeitsgemeinschaft Internistische Onkologie (FLOT65+). Eur J Cancer 49: 835-842.

24. Van Cutsem E, Boni C, Tabernero J, Massuti B, Middleton G, et al. (2015) Docetaxel plus oxaliplatin with or without fluorouracil or capecitabine in metastatic or locally recurrent gastric cancer: A randomized phase II study. Ann Oncol 26: 149-156.

25. Pfeiffer P, Qvortrup C, Krogh M, Schoennemann K, Vestermark LW, et al. (2017) S-1 in combination with docetaxel and oxaliplatin in patients with advanced gastro-esophageal adenocarcinoma: Two parallel phase 1/2a studies. Acta Oncol 56: 46-51.

26. Petrelli F, Tomasello G, Ghidini M, Passalacqua R, Barni $S$ (2017) Modified schedules of DCF chemotherapy for advanced gastric cancer: a systematic review of efficacy and toxicity. Anticancer Drugs 28: 133-141.

27. Amarantidis K, Xenidis N, Chelis L, Chamalidou E, Dimopoulos $\mathrm{P}$, et al. (2011) Docetaxel plus oxaliplatin in combination with capecitabine as first-line treatment for advanced gastric cancer. Oncology 80: 359-365.

28. Goel G, Jauhri M, Negi A, Aggarwal S (2010) Feasibility study of docetaxel, oxaliplatin and capecitabine combination regimen in advanced gastric or gastroesophageal adenocarcinoma. Hematol Oncol Stem Cell Ther 3: 55-59.

29. Stein A, Arnold D, Thuss-Patience PC, Moehler M, Grothe W, et al. (2014) Docetaxel, oxaliplatin and capecitabine (TEX regimen) in patients with metastatic gastric or gastro-esophageal cancer: Results of a multicenter phase I/ II study. Acta Oncol 53: 392-398.

30. Klastersky J, de Naurois J, Rolston K, Rapoport B, Maschmeyer G, et al. (2016) Management of febrile neutropaenia: ESMO Clinical Practice Guidelines. Ann Oncol 27: v111-v118.

31. Al-Batran SE, Hozaeel W, Jager E (2012) Combination of trastuzumab and triple FLOT chemotherapy (5-fluorouracil/leucovorin, oxaliplatin, and docetaxel) in patients with HER2-positive metastatic gastric cancer: Report of 3 cases. Onkologie 35: 505-508. 